\title{
Prevalence and determinants of cigarette smoking among adolescents in Blantyre City, Malawi
}

\author{
A.S. MUULA* \\ Department of Community Health, University of Malawi, College of Medicine, Blantyre, Malawi and Department of \\ Epidemiology, School of Public Health, University of North Carolina at Chapel Hill, North Carolina, United States
}

\begin{abstract}
Tobacco smoking is a major risk factor for non-communicable diseases such as ischaemic heart disease, stroke, chronic obstructive airways disease and several cancers. There is little data about the prevalence and determinants of smoking among adolescents in southern Africa. This study aimed to determine the prevalence and determinants of cigarette smoking among adolescents in Blantyre City, Malawi. Cross-sectional data were obtained from school-going adolescents in Blantyre in 2001 using the Global Youth Tobacco Survey data collection instrument. Data were analysed to determine prevalence of current and ever cigarette smoking, and predictors of smoking. The prevalence of current smoking and ever smoking were $3.0 \%$ and $15.6 \%$, respectively. Predictors of current tobacco smoking included male gender, having friends or parents who smoked, having been exposed to advertisements about tobacco brands on television and having seen a lot of advertisements in newspapers and magazines. School programmes that included being taught about smoking in class and a class discussion on the dangers of tobacco were not associated with reduced current smoking. Intervention programmes aiming to curb tobacco smoking among adolescents should focus on dealing also with parental smoking, peer influence and pay special attention toward male gender. School-based programmes to prevent smoking should be evaluated as some may have little impact in influencing current smoking status.
\end{abstract}

Keywords: adolescent, smoking, tobacco, determinants, Malawi

\section{Introduction}

Tobacco smoking is a risk factor for several chronic diseases such as ischaemic heart disease, hypertension, chronic obstructive airways diseases, stroke, and lung and other cancers. Tobacco firms are increasingly recruiting young people to smoke. The prevalence and associated factors related to smoking in Malawi have not been well described. Much of the research interest in the country is in infectious or communicable diseases mostly HIV/AIDS, tuberculosis and malaria. However, adolescent smoking is a recognized growing problem worldwide. In a study of seventh graders in the United States, Dowdell (2006) reported that 31\% were current smokers and $25 \%$ of these had started smoking between the ages of 11 and 13 years. An earlier study by the same author (Dowdell, 2002) reported that $43 \%$ of seventh graders had ever smoked. In Nigeria, Lawoyin et al. (2005) reported that only $1.5 \%$ of secondary school students were current tobacco smokers. Smoking, as health risk behaviour has also been associated with other risk behaviours such as alcohol use and unsafe sexual practices (Mpofu et al., 2006). Smoking among adolescents may not have adverse effects directly related to tobacco but also consequences or ancillary activities.

In Malawi, the tobacco crop contributes at least $60 \%$ of the foreign exchange earnings. Pampel (2005) has reported that adult tobacco smoking in Malawi is more common amongst males, the uneducated and urban dwellers. Overall adult smoking prevalence was estimated at $20 \%$ in males and $9 \%$ among females. While there has been some assessment of adult smoking practices in Malawi, little attention has been directed towards adolescent smoking patterns and determinants.

\section{Materials and Methods}

\section{Study site}

This study was carried out in Blantyre City in Malawi, located in the southern region. It has a population of about 700,000 inhabitants. The study was conducted among adolescents in public schools within Blantyre City. This study was conducted as an ancillary study to estimate the prevalence of, and the association factors of smoking among school going adolescents. Data was obtained from the Centers for Disease Control and Prevention, United States at the Global Youth Tobacco Survey (GYTS) Programme. Through the programme, a school-based cross-sectional survey aimed to examine tobacco use among school-going adolescents in Malawi was carried out (www.cdc.gov/ TOBACCO/Global/gyts/reports/afro/2001/ Malawi_2001.htm). While this report has described on the prevalence of smoking, the associations between current smoking status and selected sociodemographics has not been reported.

The GYTS questionnaire was self administered among eligible students in the participating schools (Global Youth Tobacco Survey Collaborative Group, 2002). Current smoking was defined as 'smoking a cigarette within the past 30 days', while 'ever smoked' was defined as having smoked tobacco even a single 
puff in one's life. Associations between several predictors for tobacco use such as gender, having friends who smoked, parents who smoked, exposure to tobacco advertisements, or having participated in a school-based anti-tobacco activity, were evaluated.

\section{Participant selection}

A two-stage cluster sampling technique was used to identify eligible public schools. In the first stage of the sampling both schools were selected with the probability of being in the sample proportional to enrolment size. Students in standards 7 and 8 and Form one in the eligible classes were invited to participate. Enrolment information had been obtained from the Ministry of Education, Science and Technology. In the second stage of sampling, classes from each of the 26 eligible schools were selected.

\section{Survey instrument}

The questionnaire comprised 56 multiple-choice questions with core items selected from the GYTS. The questionnaire has been described elsewhere (Warren et al., 2000). The questionnaire aimed to solicit responses on the following: experience with tobacco, cigarettes and other tobacco products, environmental (passive) exposure to tobacco, access to tobacco products, perception about tobacco use, exposure to tobacco-related advertisements and promotion.

The training workshops for survey coordinators and administrators were done four weeks prior to data collection, where field researchers were instructed on the standard procedures to assure comparability on data collection.

\section{Ethical considerations}

Permission to conduct the study was obtained from the Ministry of Education, Science and Technology. When a class was selected, all students in the class were eligible to participate. Students were informed that they were free not to participate and were not compelled to complete any questionnaire. Questionnaires were completed in class without the presence of a teacher.

\section{Data analysis}

Data were analysed using Stata software version 9 (Stata Corp, Texas). Logistic regression model was fitted to obtain prevalence odds ratio. An \pm level of 0.05 was chosen to obtain $95 \%$ confidence intervals.

\section{Results}

Of the 1308 study participants, 1191 (91\%) reported their ages and 117 were missing. The mean age was
$13.9(\mathrm{SD}=1.5)$ years. The ages ranged from 11 years to 17 years. There were 610 (46.6\%) females, 574 (43.9\%) males and 124 (9.5\%) missing.

Of the 1253 participants who reported their smoking status, $36(3.0 \%, 95 \%, \mathrm{CI}=2.5-3.2)$ were current smokers. A total of 204 (15.6\%) had ever smoked and $142(69.6 \%)$ reported no longer smoking. For those participants that reported to have ever smoked, 131 were males, 51 females and 22 missing. In bi-variate analysis, males were more likely to be current smokers than females, OR 2.6 (1.2-5.8). Males were also more likely to have ever smoked, OR 3.2 (2.3-4.6).

Study participants who had at least a parent smoker were also likely to be smokers themselves, OR 2.8 (1.2-6.3). Having a friend who was a smoker was strongly associated with increased tobacco use, OR, 9.7 (4.7-20.2). The association for males was OR 12.1 (4.0-36.8) and in females OR 12.8 (3.1-52.4). Having a father who was a smoker was strongly associated with the adolescent being a smoker, OR 3.5 (1.53-7.8).

Exposure to tobacco advertisement was assessed by asking about experience with television tobacco advertisements, having seen tobacco promotion in magazine and newspapers and having been contacted by a representative of a tobacco company for free cigarettes. Having been contacted by a tobacco company representative and been exposed to adverts in newspapers and magazine were not associated with current tobacco smoking, OR $0.5(0.9-4.6)$ and 1.2 (0.2-1.1) respectively. Having seen a lot of advertisements in magazines was however associated with current tobacco use, OR 2.2 (1.03-4-81). Exposure to tobacco brands on television was associated with current tobacco use, OR=4.04 (1.89.1).

An assessment was also made as to whether exposure to anti-tobacco messages in school was associated with current tobacco smoking. The odds of current smoking after having been taught about the dangers of smoking within the past year was 0.8 (0.41.6) while and having had a class discussion on tobacco was associated with current smoking with OR 0.71 (0.34-1.49) and 0.81 (0.40-1.67), respectively. Participants who believed that smoking is harmful to health and that smoking should be banned in public places were more likely to be non-current smokers, OR $0.3(0.1-0.7)$ and $0.3(0.2-0.7)$, respectively.

\section{Discussion}

This study reports a prevalence of current smoking among school-going adolescents aged between 11 and 
17 years in Blantyre, Malawi at 3.0\% and prevalence of history of having ever smoked at $15 \%$. This was slightly lower than estimates from Kampala, Uganda at 5.3\% (Mpabulungi \& Muula, 2004) but higher than that reported from Nigeria (Lawoyin et al., 2005). Our results have lower estimates compared to a study of 14-18 year olds in the United States (Rice et al., 2006) that reported that cigarette smoking in past 30 days was $6.9 \%$. In the same study, $29 \%$ of the participants in the United States study reported to have ever smoked cigarettes.

Gritz et al. (2003) reported that parental and household influences (parental education, marital status, household smoking) were important predictors of ever smoking, but not of susceptibility to smoking, among African Americans. Hispanic adolescents were significantly influenced by environmental influences, namely smoking by other household members (ever smoking) and by peers (susceptibility and ever smoking), although peer pro-tobacco influences (friends who smoke or friends' approval of smoking) were important predictors of susceptibility to smoking or ever smoking for all three ethnic groups. In our study, it was also shown that parental smoking and friends who smoke were strongly associated with being a smoker. It is possible that adolescents who have parents who smoke may perceive smoking as acceptable and may not get parental sanctions for smoking. It is also possible that availability of cigarettes within household may influence the adolescent to initiate and maintain a smoking habit. In the United States, the exposure to tobacco-related advertising was a risk factor for White (susceptibility and ever smoking) and African American (susceptibility only) adolescents but not for Hispanic adolescents (Gritz et al., 2003). In our study, having watched television advertisements was associated with current tobacco smoking. Tobacco related advertisements were associated with current smoking for participants who described themselves as having seen a 'lot of advertisements'.

This study has several limitations. Firstly, we determined current smoking status based on selfreports. Study participants may have failed to report accurately their smoking status due to a variety of reasons. We did not validate the self-reports by laboratory studies such as carbon monoxide content of exhaled smoke. Jagoe et al. (2002) in a study conducted in Ilala, Dar es Salam, Tanzania were able to reclassify $7.3 \%$ males and $27.3 \%$ of smokers as current smokers based on exhaled alveolar carbon monoxide while self-reports would have identified them as not current smokers. How much bias may have occurred in our study is not known.
Since this was a school-based study and that it captured only adolescents in schools, it missed out all those that were not in school, and hence the prevalence estimates and the determinants of tobacco use obtained in this study may therefore reflect the situation among those adolescents attending school and cannot be extrapolated to out-of-school adolescents. The fact that only public schools were eligible for participation limits the generalisability of the finding to the wider school-going population in Blantyre, Malawi.

In conclusion, intervention programmes aimed to prevent adolescent smoking should give consideration to the determinants of smoking such as gender, parental and peer influence and exposure to advertisements. A successful anti-tobacco initiative will therefore ensure that there is continuum of effort within school, at home and within the wider society.

\section{Acknowledgements}

The GYTS is a collaborative project of $\mathrm{WHO} / \mathrm{CDC} /$ participating countries. Analyses of GYTS data are not necessarily endorsed by the $\mathrm{WHO} / \mathrm{CDC} /$ participating countries. We are grateful to the following people for their assistance in this study: Ms. Leane Riley, Dr. Wick Warren, Ms. Juliette Lee, Mr Curtis Blanton (CDC) Dr. Charles Maringo and Karen Klimowski (World Health Organization, AFRO Region) and Mr. John Kapito, Consumers Association of Malawi. While the data for the study were supplied by the CDC (United States), the organization is not responsible for the analysis and did not influence decision to submit manuscript for publication.

\section{References}

Dowdell, E.B. (2002) Urban seventh graders and smoking: a health risk behaviour assessment. Issues in Comprehensive Pediatric Nursing 25, 217-229.

Dowdell, E.B. (2006) Alcohol use, smoking and feeling unsafe: health risk behaviours of two urban seventh grade classes. Issues in Comprehensive Pediatric Nursing 29,157-171.

Global Youth Tobacco Survey Collaborative Group. (2002). Global Tobacco use among youth: a cross country comparison. Tobacco Control 11, 252270.

Gritz, E.R., Prokhorov, A.V., Hudmon, K.S., MullinJones, M., Rosenblum, C., Chang, C.C., Chamberlain, R.M., Taylor, W.C., Johnston, D. $\&$ de Moor, C. (2003) Predictors of susceptibility to smoking and ever smoking: a longitudinal 
study in a triethnic sample of adolescents. Nicotine \& Tobacco Research 5, 493-506.

Jagoe, K., Edwards, R., Mugusi, F., Whiting, D. \& Unwin, N. (2002). Tobacco smoking in Tanzania, East Africa: population based smoking prevalence using alveolar carbon monoxide as a validation tool. Tobacco Control 11, 210-214.

Lawoyin, T.O., Ajumobi, O.O., Abdul, M.M., Abdul Malik, J.O., Adegoke, D.A. \& Agbedeyi, O.A. (2005) Drug use among senior secondary school students in rural Nigeria. African Journal of Medicine and Medical Sciences 34, 355-359.

Mpabulungi, L. \& Muula, A.S. (2004) Tobacco use among high school students in Kampala, Uganda: questionnaire study. Croatian Medical Journal 45, 80-83.

Mpofu, E., Caldwell, L., Smith, E., Flisher, A.J., Matthews, C., Wegner, L., Vergnani, T. (2002)
Rasch modeling of the structure of health risk behaviour in South African adolescents. Journal of Applied Measurements 7, 323-334.

Pampel, F.C. (2005) Patterns of tobacco use in the early epidemic stages: Malawi and Zambia, 2000-2002. American Journal of Public Health 95, 1009-1015.

Rice, V.H., Weglick, L.S., Templin, T., Hammad, A., Jamil, H. \& Kulwicki, A. (2006) Predictors of Ara American Adolescent Tobacco use. Merrill Palmer Quarterly (Wayne State University Press) 52, 327-342.

Warren, C.W., Riley, L., Asma, S., Eriksen, M.P., Green, L., Blanton, C., Loo, C., Batchelor S. \& Yach, D. (2000) Tobacco use by youth: a surveillance report from the Global Youth Tobacco Survey Project. Bulletin of the World Health Organization 78, 868-876. 